\title{
On the Configuration Space of Planar Closed Kinematic Chains
}

\author{
Gerhard Zangerl \\ (Communicated by Gudrun Albrecht)
}

\begin{abstract}
A planar kinematic chain consists of $n$ links connected by joints. In this work we investigate the space of configurations, described in terms of joint angles, that guarantee that the kinematic chain is closed. We give explicit formulas expressing the joint angles that guarantee closedness by a new set of parameters. Moreover, it turns out that these parameters are contained in a domain that possesses a simple structure. We expect that the new insight can be applied for several issues such as motion planning for closed kinematic chains or singularity analysis of their configuration spaces. In order to demonstrate practicality of the new method we present numerical examples.
\end{abstract}

Keywords: configuration space; closed kinematic chain; path planning.

AMS Subject Classification (2020): Primary: 93C35 ; Secondary: 93C57; 93C99.

\section{Introduction}

In this work we investigate the configuration space of closed planar kinematic chain (CKC) with $n$ links connected by revolute joints in terms of its joint angles. In many fields like robotics computational biology or protein kinematics it is of immense interest to understand the configuration space of a CKC. For instance, in robotics the problem to connect a start, $\alpha_{s}$, and goal configuration, $\alpha_{g}$ naturally appears and thus requires knowledge of the configuration space, which is typically a manifold or variety in the ambient space formed by the robots joint variables. The configuration space is even more complicated if additional constraints like obstacle, link-link avoidance, or limited joint angles are included. Two main strategies, probabilistic and geometric approaches, to investigate configuration spaces have been developed so far.

Probabilistic methods have been successfully applied for constrained motion planning. They are especially important in practical situations with high dimensions that include complex constraints such as obstacle avoiding. Typically these methods are based on the generation of random configurations in ambient joint space followed by a check up if they approximately satisfy the desired constrains. Repeating this procedure results in a discrete version of the configuration space that is very useful in applications. Probabilistic methods have been applied in different situations, which can be found in $[1,4,13,6,7,18,17]$,

Besides the approaches using randomness other works focused on questions about the geometry and topology of the configuration spaces of kinematic chains. Insight about the global geometry of configuration spaces is very important in applications. Early discoveries have been made by [14, 3]. In their fundamental work Kapovitch and Milgram established important results about the geometry, which led to novel path planning algorithms. For instance in [11,12] it is used that the configuration space of a CKC consists of two connected components when it possess three long links. An application of this result is that path planning can be done easily for this special kind of CKC's. Also for the more difficult case, when CKC's do not have three long links algorithms were derived in [11,12]. They also developed path planners in the case of $p$ point obstacles in the plane [15]. Another geometric approach was recently recognized by Han, Rudolph and Blumenthal. They 
discovered that it is very beneficial to describe the configuration space of CKC by different parameters than the joint angles, see $[8,10,9]$. Their idea is to use the length of diagonals from the positions of revolute joints to the origin $O$ as depicted on the right side of Figure 2. It turns out that for a CKC the length of these diagonals can be computed as solution of a system of linear inequalities, which means that all feasible diagonal lengths can be described by a convex polyhedron that can be handled by methods of linear programming [16]. Given feasible diagonal lengths, several configurations of the CKC can be constructed, since each link of the chain can be flipped over a diagonal. Thus in $[8,10]$ any configuration can be obtained from a set of diagonals and a vector that represents the choices of flipping, which shows that the configuration space is formed by several copies of the polyhedron given by the system of inequalities. This practically convex structure is very useful for motion planning. In $[9,10]$ paths between CKC with 1000 links are computed very efficiently.

Contribution of this work: We develop a new method that explicitly computes configurations of a CKC with $n$ links, which are described by its joint angles. Compared to other methods it does not require linear programming to solve a system of linear inequalities like in $[8,10]$ nor does it rely on probabilistic principles. More precisely, it turns out that a configuration can be computed from new parameters contained in a very simple domain, namely a $n-3$ dimensional cube. The developed method can be used to easily sample configuration space of a CKC and thus is expected to be useful in practical applications.

Outline of this text: In section 2 we give a mathematical description of a CKC and its configuration space. Then the basic algorithm that explicitly describes how configurations of a CKC can be computed is developed in section 2. In section 3.1 we describe the set of new parameters and show how they can be used to compute a vector of joint angles of a CKC. Finally, we give numerical examples that show the validity of the developed method.

\section{Configuration space}

To describe the configuration space of a CKC with link lengths $a_{1}, \ldots, a_{n}$ we introduce Cartesian coordinates in two dimensional Euclidean space. Moreover we place one of the links of the CKC so that it is supported by the positive $x$-axis and so that one of its ends coincides with the origin. Without loss of generality we can assume that the link $a_{n}$ of the chain is fixed in the described manner, see Figure 1. In the following, we identify an angle $\alpha$ with its corresponding point on $S^{1}$. Further, for $1 \leq k \leq n$ and a vector of angles $\alpha^{k}:=\left(\alpha_{1}, \ldots, \alpha_{k}\right) \in\left(S^{1}\right)^{k}$ we denote by

$$
f_{a, k}:\left(S^{1}\right)^{k} \rightarrow \mathbb{R}^{2}, \quad f_{a, k}\left(\alpha^{k}\right)=\sum_{j=1}^{k} a_{j}\left(\begin{array}{c}
\cos \left(\alpha_{j}\right) \\
\sin \left(\alpha_{j}\right)
\end{array}\right) .
$$

the $k$-th endpoint map of a kinematic chain, where $a=\left(a_{1}, \ldots, a_{n}\right)$ is the vector of link lengths. We will call $\alpha^{n-1}$ a configuration of the CKC with link lengths $a_{1}, \ldots, a_{n}$ if it satisfies the closure condition, which means that it is contained in the set

$$
\mathrm{C}_{a}^{\mathrm{onv}}=\left\{\alpha^{n-1} \in\left(S^{1}\right)^{n-1}: f_{a, n-1}\left(\alpha^{n-1}\right)=\left(\begin{array}{c}
a_{n} \\
0
\end{array}\right)\right\}=f_{a, n-1}^{-1}\left(a_{n}, 0\right) .
$$

If no restrictions on the endpoint map are imposed $\alpha^{n-1}$ will just be called a configuration of the kinematic chain (KC) with $n-1$ links.

Furthermore, the analysis in this work uses the simple observation that it is sufficient to understand the space

$$
\mathcal{C C}_{a}^{o n v}=\left\{\beta^{n-1} \in\left(S^{1}\right)^{n-1}:\left\|f_{a, n-1}\left(\beta^{n-1}\right)\right\|_{2}^{2}=a_{n}^{2}\right\}
$$

in order to describe $C_{a}$, where $\|\cdot\|_{2}$ denotes the Euclidean norm. From the definition of $\mathcal{C C}_{a}^{o n v}$ it is clear that any configuration $\beta^{n-1} \in \mathcal{C C}_{a}^{o n v}$ satisfies that its endpoint

$$
f_{a, n-1}\left(\beta^{n-1}\right) \in K_{a_{n}}
$$

lies on the circle $K_{a_{n}}$ that is centred on the origin and has radius $a_{n}$. We will say that $\beta^{n-1}$ is closed up to a rotation and call it a circular configuration of a CKC. Clearly, any circular configuration $\beta^{n-1}$ can be rotated by 


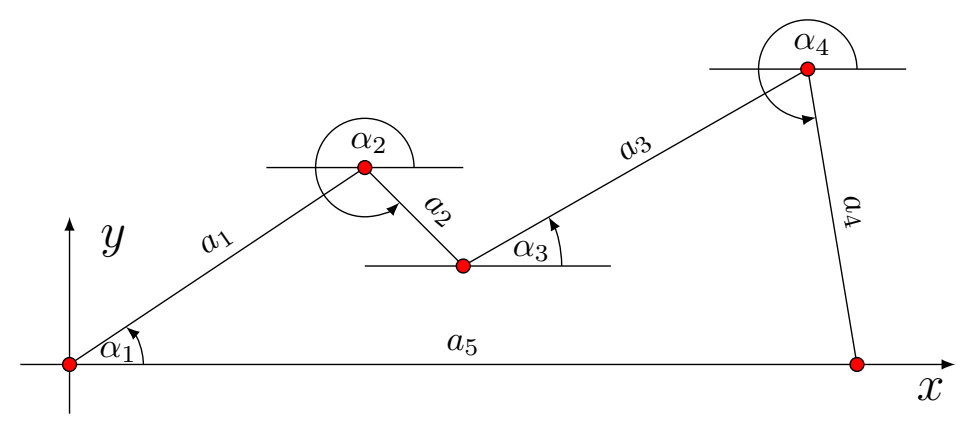

Figure 1. A CKC with $n=5$ five links. The link $a_{5}$ is supported on the positive $x$-axis and one of its ends coincides with the origin

an angle $\lambda$,

$$
\beta^{n-1}+\lambda:=\left(\beta_{1}+\lambda, \ldots, \beta_{n-1}+\lambda\right),
$$

so that $\beta^{n-1}+\lambda \in C_{a}$. Thus, if we are able to give an efficient method to compute the set of solutions to the implicit equation

$$
\left\|f_{a, n-1}\left(\beta^{n-1}\right)\right\|_{2}^{2}=a_{n}^{2}
$$

we also obtain configurations in $C_{a}$ by the following two step algorithm:

(i) Compute a circular configuration $\beta^{n-1} \in \mathcal{C C}_{a}^{o n v}$

(ii) Determine $\lambda$ such that $\alpha^{n-1}=\beta^{n-1}+\lambda \in \mathrm{C}_{a}^{\text {onv }}$

Once a circular configuration is obtained step (ii) is a rather simple task. Therefore, in the following we will focus on the solution of step (i). This step is based on the fact that the trigonometric equation (2.4), which in its expanded form is given as

$$
\sum_{i=1}^{n-1} a_{i}^{2}+2 \sum_{i<j}^{n-1} a_{i} a_{j} \cos \left(\beta_{i}-\beta_{j}\right)=a_{n}^{2}
$$

allows for some kind of backwards substitution, see section 2.2. By the preimage theorem we know that the set of all circular configurations of a CKC with $n$ links satisfying (2.5) is a manifold of dimension $n-2$, whenever $a_{n}^{2}$ is a regular value of the map $g\left(\beta^{n-1}\right):=\left\|f_{a, n-1}\left(\beta^{n-1}\right)\right\|_{2}^{2}$. In all other cases the space $\mathcal{C C}_{a}^{\text {onv }}$ may have singular points.

\subsection{Mathematical tools and notations}

Surprisingly, the trigonometric equation (2.5) can be rearranged into an equation of the same type but with one joint angle less appearing on its left hand side. For the computations we use that a linear combination of sine and cosine functions can be written as

$$
a \sin (x)+b \cos (x)=c \sin (x+\varphi(a, b)),
$$

where $c=\sqrt{a^{2}+b^{2}}$ and $\varphi(a, b)=$ atan2 $(b, a)$ is the function described in Figure 2.

In order to achieve a compact presentation of the results that will follow it is important to introduce abbreviations. For this purpose consider

$$
a_{n-1} \sum_{j=1}^{n-2} a_{j} \cos \left(\beta_{n-1}-\beta_{j}\right)+\sum_{i<j}^{n-2} a_{i} a_{j} \cos \left(\beta_{i}-\beta_{j}\right)=\frac{a_{n}^{2}-\sum_{i=1}^{n-1} a_{i}^{2}}{2},
$$



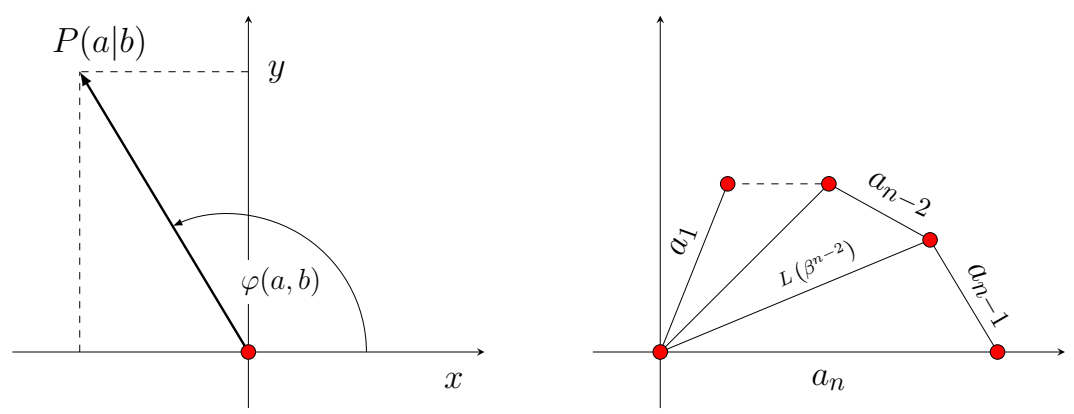

Figure 2. Left: The function atan2 gives the angle between the $x$-axis and the vector from the origin to $P(a \mid b)$. Right: A circular configuration with endpoint $\left(a_{n}, 0\right)$. The picture shows anchored diagonals of the CKC

which is an equivalent form of (2.5) that is obtained by fixing an index to be $n-1$ and rearranging the remaining terms. Finally, using trigonometric summation formulas we arrive at

$$
\begin{aligned}
& a_{n-1} \sin \left(\beta_{n-1}\right) \sum_{j=1}^{n-2} a_{j} \sin \left(\beta_{j}\right)+a_{n-1} \cos \left(\beta_{n-1}\right) \sum_{j=1}^{n-2} a_{j} \cos \left(\beta_{j}\right)+ \\
& \sum_{i<j}^{n-2} a_{i} a_{j} \cos \left(\beta_{i}-\beta_{j}\right)=\frac{a_{n}^{2}-\sum_{i=1}^{n-1} a_{i}^{2}}{2}
\end{aligned}
$$

In the last expression addition formula (2.6) can be applied, which motivates the following abbreviations:

For a CKC with link lengths $a_{1}, a_{2}, \ldots, a_{n}$, a circular configuration $\beta^{n-1}=\left(\beta_{1}, \ldots, \beta_{n-1}\right)$ and for $1 \leq k \leq n-1$ we denote by $\beta^{n-k}:=\left(\beta_{1}, \ldots, \beta_{n-k}\right)$ and we write $S_{n-k}=\sum_{i=1}^{n-k} a_{i}^{2}$ for the sum of the squared link lengths. Furthermore, for $\beta^{n-k}$ we abbreviate terms

$$
\begin{aligned}
X\left(\beta^{n-k}\right) & :=\sum_{i<j}^{n-k} a_{i} a_{j} \cos \left(\beta_{i}-\beta_{j}\right), \\
\Phi\left(\beta^{n-k}\right) & :=\varphi\left(\sum_{j=1}^{n-k} a_{j} \cos \left(\beta_{j}\right), \sum_{j=1}^{n-k} a_{j} \sin \left(\beta_{j}\right)\right),
\end{aligned}
$$

which naturally appear, when applying formula (2.6) for equation (2.7). With this shortened notation an important quantity for a CKC, its diagonal length, which is the distance of $f_{a, n-k}\left(\beta^{n-k}\right)$ and the origin, can be expressed as

$$
L\left(\beta^{n-k}\right):=\sqrt{S_{n-k}+2 X\left(\beta^{n-k}\right)} .
$$

Note that we assume $\beta^{1}=\beta_{1}$ and $X\left(\beta^{1}\right)=0$. Finally, for computations carried out in 2.2 it is convenient to denote by $C^{k}:=\left(C_{3}, \ldots, C_{k}\right)$ a vector with $k-2$ entries for $3 \leq k \leq n-1$ and let $C_{n}$ be the constant given by $C_{n}:=\left(a_{n}^{2}-S_{n-1}\right) / 2$, which is the right hand side of equation (2.7).

\subsection{Method for the Computation of Circular Configurations}

We give a new method to obtain solutions to equation (2.5). In the proof of Theorem 2.1 a procedure is described how solutions are obtained by reducing the length of the CKC step by step. For $k \geq 1$ the solution method involves the choice of a real value $C_{n-k}$ between the roots of a quadratic equation $\mathrm{E}_{k}\left(C_{n-k+1}\right)$ corresponding to the value $C_{n-k+1}$, see Definition (A.1). This value $C_{n-k}$ then defines again a quadratic equation $\mathrm{E}_{k}\left(C_{n-k}\right)$ and a new value $C_{n-k+1}$ is chosen between its roots and so on. In order to guarantee that this procedure is well defined, we need that all quadratic equations connected in this manner have real solutions, which is guaranteed by Lemma A.1 in the appendix. 
In the following Theorem we will work with equation

$$
\sum_{i<j}^{n-1} a_{i} a_{j} \cos \left(\beta_{i}-\beta_{j}\right)=C_{n},
$$

which is just a rearranged form of (2.5). Also abbreviations introduced in section 2.1 appear quite naturally in the proof of the Theorem.

Theorem 2.1 (Computation of circular configurations). Circular configurations $\beta^{n-1} \in \mathcal{C C}_{a}^{\text {onv }}$, that is a configuration satisfying the equation (2.8) can be obtained by the following procedure:

1. Compute a vector $C^{n-1}=\left(C_{3}, \ldots, C_{n-1}\right)$ with entries satisfying

$$
C_{n-k+1}^{-} \leq C_{n-k} \leq C_{n-k+1}^{+}, \quad \text { and } \quad C_{n-k}^{\min } \leq C_{n-k} \leq C_{n-k}^{\max }
$$

for $1 \leq k \leq n-3$, where

$$
C_{n-k+1}^{ \pm}=C_{n-k+1}+a_{n-k}^{2} \pm a_{n-k} \sqrt{2 C_{n-k+1}+S_{n-k}} .
$$

and $C_{n-k}^{\min }, C_{n-k}^{\max }$ denote the maximal and minimal values that $X\left(\delta^{n-k-1}\right)$ can take on for $\delta^{n-1} \in\left(S^{1}\right)^{n-1}$.

2. For $1 \leq k \leq n-2$ compute $\beta_{n-k}$ according to the equation (where $C_{2}:=0$ and $\beta^{1}:=\beta_{1}$ )

$$
a_{n-k} \sin \left(\beta_{n-k}+\Phi\left(\beta^{n-k-1}\right)\right)=\frac{C_{n-k+1}-C_{n-k}}{\sqrt{S_{n-k-1}+2 C_{n-k}}},
$$

whenever the denominator of the term on the right hand side is not zero. Otherwise the angle $\beta_{n-k}$ can be chosen arbitrarily.

Then $\beta^{n-1}=\left(\beta_{1}, \ldots, \beta_{n-1}\right) \in \mathcal{C C}_{a}^{o n v}$.

Proof. Assume $\beta^{n-1}$ solves equation (2.8), which due to (2.7) and the abbreviations in section 2.1 can be written as

$$
\begin{aligned}
& a_{n-1} \sin \left(\beta_{n-1}\right) \sum_{j=1}^{n-2} a_{j} \sin \left(\beta_{j}\right)+a_{n-1} \cos \left(\beta_{n-1}\right) \sum_{j=1}^{n-2} a_{j} \cos \left(\beta_{j}\right)+ \\
& X\left(\beta^{n-2}\right)=C_{n} .
\end{aligned}
$$

The latter equation is a linear combination of $\sin \left(\beta_{n-1}\right)$ and $\cos \left(\beta_{n-1}\right)$ and thus can further be simplified to

$$
\begin{aligned}
& \sqrt{\left(\sum_{j=1}^{n-2} a_{j} \sin \left(\beta_{j}\right)\right)^{2}+\left(\sum_{j=1}^{n-2} a_{j} \cos \left(\beta_{j}\right)\right)^{2}} a_{n-1} \sin \left(\beta_{n-1}+\Phi\left(\beta^{n-2}\right)\right)+ \\
& X\left(\beta^{n-2}\right)=C_{n} .
\end{aligned}
$$

Expanding the squares shows that this is equivalent to

$$
a_{n-1} \sin \left(\beta_{n-1}+\Phi\left(\beta^{n-2}\right)\right) \sqrt{S_{n-2}+2 X\left(\beta^{n-2}\right)}+X\left(\beta^{n-2}\right)=C_{n},
$$

where the square root is just given by the abbreviation $L\left(\beta^{n-2}\right)$. If $L\left(\beta^{n-2}\right)=0$ we have that $X\left(\beta^{n-2}\right)=C_{n}$ has to be satisfied and $\beta_{n-1}$ is an arbitrary value. Otherwise, rewriting the latter equation gives

$$
a_{n-1} \sin \left(\beta_{n-1}+\Phi\left(\beta^{n-2}\right)\right)=\frac{C_{n}-X\left(\beta^{n-2}\right)}{\sqrt{S_{n-2}+2 X\left(\beta^{n-2}\right)}} .
$$

Since the latter equation can be solved for $\beta_{n-1}$ the right hand side satisfies

$$
-a_{n-1} \leq \frac{C_{n}-X\left(\beta^{n-2}\right)}{\sqrt{S_{n-2}+2 X\left(\beta^{n-2}\right)}} \leq a_{n-1},
$$


which is the case when $X\left(\beta^{n-2}\right)$ is equal to a number $C_{n-1}$ that is contained within the roots $C_{n}^{ \pm}$of the quadratic equation

$$
\left(C_{n}-C\right)^{2}-a_{n-1}^{2}\left(S_{n-2}+2 C\right)=0,
$$

and when $C^{\min } \leq C_{n-1} \leq C^{\max }$ holds. Note that the roots of the latter equation are real by lemma A.1. Thus we have that $\beta^{n-2}$ satisfies the equation

$$
X\left(\beta^{n-2}\right)=\sum_{i<j}^{n-2} a_{i} a_{j} \cos \left(\beta_{i}-\beta_{j}\right)=C_{n-1},
$$

which is of the same Type as (2.8). Consequently, the computations from above can be repeated and after $k$ times we end up with

$$
a_{n-k} \sin \left(\beta_{n-k}+\Phi\left(\beta^{n-k-1}\right)\right)=\frac{C_{n-k+1}-X\left(\beta^{n-k-1}\right)}{\sqrt{S_{n-k-1}+2 X\left(\beta^{n-k-1}\right)}} .
$$

Again, if $L\left(\beta^{n-k}\right) \neq 0$, the latter equation can be solved for the $\beta_{n-k}$ and thus $X\left(\beta^{n-k-1}\right)$ equals a value $C_{n-k}$ that satisfies $C_{n-k}^{\min } \leq C_{n-k} \leq C_{n-k}^{\max }$ and which is contained within the roots $C_{n-k+1}^{ \pm}$of the equation

$$
\left(C_{n-k+1}-C\right)^{2}-a_{n-k}^{2}\left(S_{n-k-1}+2 C\right)=0,
$$

given by (2.10). Thus a circular configuration $\beta^{n-1} \in \mathcal{C C}_{a}^{o n v}$ defines values $C_{n-k}$ satisfying the system of inequalities (2.9). Conversely, if we have a solution to the systems of inequalities (2.9) a circular configuration $\beta^{n-1} \in \mathcal{C C}_{a}^{o n v}$ can be defined according to (2.11).

The $C^{n-3}$, which entries $C_{n-k}$ are recursively obtained by the system of inequalities (2.9) form a domain in $n-3$ dimensional real space. By the proof of the last Theorem it is clear that the parameters $C_{n-k}$ are closely related to the abbreviations $L\left(\beta^{n-k-1}\right)$ introduced in section 2.1. More precisely, for $\beta \in \mathcal{C C}_{a}^{o n v}$ the term $L\left(\beta^{n-k}\right)$ is the length of the line segment connecting the origin with the endpoint $f_{a, n-k}\left(\beta^{n-k}\right)$, which we will call a diagonal of a CKC according to [8], see Figure 2. In the appendix A the relation between $C_{n-k}$ and the diagonals of a CKC is explained in more detail. However, the connection between the $C_{n-k}$ and the diagonals of a CKC motivates the following definition.

Definition 2.1 (Domain of Semi-Diagonals). We will denote the set given by

$$
\mathcal{S D}_{a}:=\left\{C^{n-1} \in \mathbb{R}^{n-3}: C_{n-k} \in\left[C_{n-k+1}^{-}, C_{n-k+1}^{+}\right], 1 \leq k \leq n-3\right\}
$$

as semi-diagonal parameters of a CKC with links $a$.

According to Theorem 2.1 from any $C^{n-3} \in \mathcal{S D}_{a} \cap Q_{a}$, where

$$
Q_{a}=\left\{C^{n-3} \in \mathbb{R}^{n-3}: C_{n-k}^{\min } \leq C_{n-k} \leq C_{n-k}^{\max }\right\},
$$

circular configurations can be computed by solving (2.11). Note, that $C_{n-k}^{\max }=\sum_{i<j}^{n-k} a_{i} a_{j}$ and $C_{n-k}^{\min }=$ $\min _{\beta} X\left(\beta^{n-k-1}\right)$ can be easily computed. Since the solution of (2.11) for a $\beta_{n-k}$ is not unique, an element $C^{n-1} \in \mathcal{S D}_{a} \cap Q_{a}$ will yield several circular configurations and we will consider all possible configurations that can be obtained from it in section 3.2. In the following section we will further investigate the set $\mathcal{S} \mathcal{D}_{a}$. It will turn out, that it can be described in a very easy way, after a substitution of variables and thus also leads to an easy description for $\mathcal{S D}_{a} \cap Q_{a}$.

\section{Further Analysis of Circular Configurations}

In this section we will study the domain $\mathcal{S D}_{a}$, which naturally appears when we compute circular configurations, in more detail. Moreover, we will have a closer look on the second step of Theorem 2.1, which requires solving equation (2.11) for an angle $\beta_{n-k}$. 


\subsection{The domain of Semi-Diagonals}

We investigate the space $\mathcal{S} \mathcal{D}_{a}$ of Semi-Diagonals further. We recall that for a CKC with $n$ links $a_{1}, a_{2}, \ldots, a_{n}$ the space $\mathcal{S} \mathcal{D}_{a}$ is defined by the tuples $C^{n-3}$, which entries satisfy the system

$$
C_{n-k+1}^{-} \leq C_{n-k} \leq C_{n-k+1}^{+}
$$

of inequalities, where

$$
C_{n-k+1}^{ \pm}=C_{n-k+1}+a_{n-k}^{2} \pm a_{n-k} \sqrt{2 C_{n-k+1}+S_{n-k}}
$$

for $1 \leq k \leq n-3$. We will see that this system can be transformed into another system after a substitution of variables, which can then be easily parametrized by a map that is defined on the $n-3$ dimensional unit cube $I^{n-3}=[-1,1]^{n-3}$.

Theorem 3.1. Let $C^{n-1} \in \mathcal{S D}_{a}$ and define new parameters $U_{n-k}$ by

$$
C_{n-k}=U_{n-k}+C_{n-k+1}+a_{n-k}^{2},
$$

for $1 \leq k \leq n-3$. Moreover we set $U_{n}:=C_{n}$ and $U^{n-1}=\left(U_{3}, \ldots, U_{n-1}\right)$. Then the entries $U_{n-k}$ satisfy the new system of inequalities

$$
-a_{n-k} \sqrt{T_{k}\left(U_{n}, \ldots, U_{n-k+1}\right)} \leq U_{n-k} \leq a_{n-k} \sqrt{T_{k}\left(U_{n}, \ldots, U_{n-k+1}\right)},
$$

where

$$
T_{k}\left(U_{n}, \ldots, U_{n-k+1}\right)=2 \sum_{j=1}^{k-1} U_{n-j}+\sum_{j=0}^{k-1} a_{n-j}^{2}, \quad \text { for } 1 \leq k \leq n-3 .
$$

Note that the first sum on the right hand side is zero in the case $k=1$ and thus $t_{1}=t_{1}\left(U_{n}\right)=a_{n}^{2}$.

Proof. We will apply (3.2) to the right hand side of system (3.1) only, since the computations for the left hand side are analogous. If we apply (3.2) we obtain

$$
U_{n-k}+C_{n-k+1}+a_{n-k}^{2} \leq C_{n-k+1}+a_{n-k}^{2} \pm a_{n-k} \sqrt{2 C_{n-k+1}+S_{n-k}},
$$

which is equivalent to

$$
U_{n-k} \leq a_{n-k} \sqrt{2 C_{n-k+1}+S_{n-k}} .
$$

If we apply the substitution (3.2) repeatedly on the right hand side we end up with

$$
\begin{aligned}
U_{n-k} & \leq a_{n-k} \sqrt{2\left(U_{n}+\sum_{j=1}^{k-1} U_{n-j}+\sum_{j=1}^{k-1} a_{n-j}^{2}\right)+S_{n-k}} \\
& \leq a_{n-k} \sqrt{2 U_{n}+2 \sum_{j=1}^{k-1} U_{n-j}+\sum_{j=1}^{k-1} a_{n-j}^{2}+S_{n-1}} \\
& \leq a_{n-k} \sqrt{2 \sum_{j=1}^{k-1} U_{n-j}+\sum_{j=0}^{k-1} a_{n-j}^{2}},
\end{aligned}
$$

where the expression under the square root is the abbreviation $T_{k}\left(U_{n}, \ldots, U_{n-k+1}\right)$ in (3.4).

We will denote the space of parameters $U^{n-1}$ introduced in Theorem 3.1 by $\mathcal{S D \mathcal { U } _ { a }}$. It is clear that any $U^{n-1} \in$ $\mathcal{S D U}$ yields a unique $C^{n-1} \in \mathcal{S} \mathcal{D}_{a}$ and vice versa, since (3.2) defines an affine map from $\mathrm{A}_{a}: \mathcal{S D \mathcal { U } _ { a }} \rightarrow \mathcal{S D}_{a}$. We will further investigate the domain $\mathcal{S D U} \mathcal{U}_{a}$ defined by the system of inequalities (3.3). It turns out that $\mathcal{S D U}{ }_{a}$ is the image of a map that is defined on the unit cube, which clearly is a nice representation for the parameters $U_{n-k}$. 
Theorem 3.2. Let $I^{n-3}$ be the $n-3$ dimensional unit cube and define the $k$-tuple $s^{k}:=\left(s_{1}, \ldots, s_{k}\right)$ for $1 \leq k \leq n-3$. Then

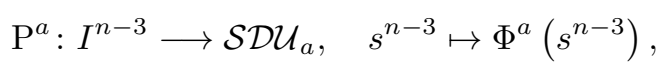

where the components of $\mathrm{P}^{a}$ are given by

$$
\begin{gathered}
\mathrm{P}_{3}^{a}\left(s^{n-3}\right)=s_{n-3} a_{3} \sqrt{T_{n-3}\left(U_{n}, \mathrm{P}_{n-1}^{a}\left(s^{1}\right), \ldots, \mathrm{P}_{4}^{a}\left(s^{n-4}\right)\right)} \\
\vdots \\
\mathrm{P}_{n-2}^{a}\left(s^{2}\right)=s_{2} a_{n-2} \sqrt{T_{2}\left(U_{n}, \mathrm{P}_{n-1}^{a}\left(s^{1}\right)\right)} \\
\mathrm{P}_{n-1}^{a}\left(s^{1}\right)=s_{1} a_{n-1} \underbrace{\sqrt{T_{1}\left(U_{n}\right)}}_{a_{n}}
\end{gathered}
$$

maps the unit cube onto the space $\mathcal{S D U}_{a}$.

Proof. We have to show that $U_{n-k}:=\mathrm{P}_{k}^{a}\left(s^{n-k}\right)$ satisfy the system of inequalities (3.3), which are equivalent to

$$
\left|U_{n-k}\right| \leq a_{n-k} \sqrt{T_{k}\left(U_{n}, \ldots, U_{n-k+1}\right)} .
$$

Note that the right side of this inequality does not depend on $U_{n-k}$, but only on $U_{n} \ldots U_{n-k+1}$. Thus plugging in

$$
\mathrm{P}_{n-k}^{a}\left(s^{k}\right)=s_{k} a_{n-k} \sqrt{T_{k}\left(U_{n}, \ldots, \mathrm{P}_{n-k+1}^{a}\left(s^{n-k+1}\right)\right)}
$$

we obtain

$$
\left|s_{k} a_{n-k} \sqrt{T_{k}\left(U_{n}, \ldots, \mathrm{P}_{n-k+1}^{a}\left(s^{n-k+1}\right)\right)}\right| \leq a_{n-k} \sqrt{T_{k}\left(U_{n}, \ldots, \mathrm{P}_{n-k+1}^{a}\left(s^{n-k+1}\right)\right)},
$$

which clearly is satisfied for $s_{k} \in[-1,1]$. Thus system (3.3) is satisfied. Conversely, if $U^{n-1} \in \mathcal{S D \mathcal { U }}$ values $s_{k}$ for $1 \leq k \leq n-3$ can easily be obtained according to Theorem (3.2). Thus we have that $\mathrm{P}^{a}\left(I^{n-3}\right)=\mathcal{S D U} \mathcal{U}_{a}$

We will illustrate the assertions of Theorems (3.1) and (3.2) by an example.

Example 3.1 (CKC with five links). We consider the CKC with equal links $a_{i}=1$ for $1 \leq i \leq 5$. In this case the inequalities defining $\mathcal{S D U} \mathcal{U}_{a}$ are given by

$$
-1 \leq U_{4} \leq 1 \quad \text { and } \quad-\sqrt{2 U_{4}+2} \leq U_{3} \leq \sqrt{2 U_{4}+2} .
$$

Then the map $\mathrm{P}^{a}:[-1,1]^{2} \rightarrow \mathcal{S D \mathcal { U } _ { a }}$ is given by

$$
s^{2}:=\left(\begin{array}{c}
s_{1} \\
s_{2}
\end{array}\right) \mapsto\left(\begin{array}{c}
s_{1} \\
s_{2} \sqrt{2 s_{1}+2}
\end{array}\right)=\left(\begin{array}{c}
U_{4}\left(s_{1}, s_{2}\right) \\
U_{3}\left(s_{1}, s_{2}\right)
\end{array}\right) .
$$

Note that $\mathrm{P}^{a}$ is injective for $s_{1} \neq-1$. Parameters $U_{3}, U_{4}$ are related to $C_{3}, C_{4}$ by the map $\mathrm{A}_{a}$ given by the equations

$$
C_{4}=U_{4}-\frac{1}{2} \quad \text { and } \quad C_{3}=U_{3}+U_{4}+\frac{1}{2}
$$

which are derived from (3.5) for $n=5$. In terms of the parameters $s^{2}=\left(s_{1}, s_{2}\right)$ we have $C_{4}=s_{1}-\frac{1}{2}$ and $C_{3}=s_{2} \sqrt{2 s_{1}+2}+s_{1}+\frac{1}{2}$

The map $\mathrm{P}^{a}$ gives a nice description for parameters $U_{n-k}$ satisfying the system of inequalities (3.3), namely by a cube. Using the affine transform $A_{a}$ we obtain a map $A_{a} \circ \mathrm{P}^{a}: I^{n-3} \rightarrow \mathcal{S D}_{a}$, which gives $\mathcal{S D \mathcal { U }}$. Points in $\mathcal{S D U}_{a} \cap Q_{a}$ can easily obtained, when restrictions $C_{n-k}^{\min } \leq C_{n-k} \leq C_{n-k}^{\max }$ are taken into account. Further investigations of $\mathrm{P}^{a}$ like its injectivity or its singularities are an interesting topic for future research. 


\subsection{Flipping over lines through diagonals of a $C K C$}

Given a $C^{n-1} \in \mathcal{S D}_{a} \cap Q_{a}$ the angle $\beta_{n-k}$ is computed from

$$
a_{n-k} \sin \left(\beta_{n-k}+\Phi\left(\beta^{n-k-1}\right)\right)=\frac{C_{n-k+1}-X\left(\beta^{n-k-1}\right)}{\sqrt{S_{n-k-1}+2 X\left(\beta^{n-k-1}\right)}} .
$$

according to Theorem 2.1. Clearly, solving for $\beta_{n-k}$ is not unique. Each time we solve for $\beta_{n-k}$ we have to choose which pre image we will take. In the following let $\varepsilon^{k}=\left(\varepsilon_{2}, \ldots, \varepsilon_{k}\right) \in\{0,1\}^{k-1}$ be a vector that contains the information, which pre images haven been chosen. We will refer to $\varepsilon^{k}$ as orientation vector. More precisely we set,

$$
\beta_{n-k}^{\varepsilon_{n-k}}=\pi \varepsilon_{n-k}+(-1)^{\varepsilon_{n-k}} S_{n, k}\left(C^{n-1}\right)-\Phi\left(\beta^{n-k-1, \varepsilon^{n-k-1}}\right) .
$$

for $1 \leq k \leq n-2$. Here the superscript $\varepsilon_{n-k}$ in $\beta_{n-k}^{\varepsilon_{n-k}}$ indicates, which preimage is chosen according to equation (3.6). Note, that we used the abbreviation

$$
S_{n, k}\left(C^{n-1}\right)=\arcsin \left(\frac{C_{n-k+1}-C_{n-k}}{a_{n-k} \sqrt{S_{n-k-1}+2 C_{n-k}}}\right)
$$

for $C^{n-1} \in \mathcal{S} \mathcal{D}_{a} \cap Q_{a}$ and the notation

$$
\beta^{n-k, \varepsilon^{n-k}}=\left(\beta_{1}, \beta_{2}^{\varepsilon_{2}}, \ldots, \beta_{n-k}^{\varepsilon_{n-k}}\right),
$$

to indicate the choice of preimages.

Therefore, for each $C^{n-1} \in \mathcal{S D}_{a} \cap Q_{a}$ and a $\varepsilon \in\{0,1\}^{n-2}$ we obtain a circular configuration

$$
\beta^{n-1, \varepsilon^{n-1}}=\left(\beta_{1}, \beta_{2}^{\varepsilon_{2}}, \ldots, \beta_{n-1}^{\varepsilon_{n-1}}\right)
$$

by formula (3.6). Note that the angle $\beta_{1}$ does note have a superscript since $\beta_{1}$ is chosen arbitrarily in the last step of (2.11) for $k=n-3$. Each $C^{n-1} \in \mathcal{S} \mathcal{D}_{a} \cap Q_{a}$ yields $2^{n-2}$ circular configurations which corresponds to the possible choices for the components of $\varepsilon^{n-1}$. There is a geometric interpretation for the value $\varepsilon_{n-k}$ in equation (3.6). It describes how the link $a_{n-k}$ is flipped over the line running through the origin and $f\left(\beta^{n-k-1, \varepsilon^{n-k-1}}\right)$, when it is attached to $f\left(\beta^{n-k-1, \varepsilon^{n-k-1}}\right)$, see Figure 3.2. Choosing the value $\varepsilon_{n-k}$ corresponds to the choice of

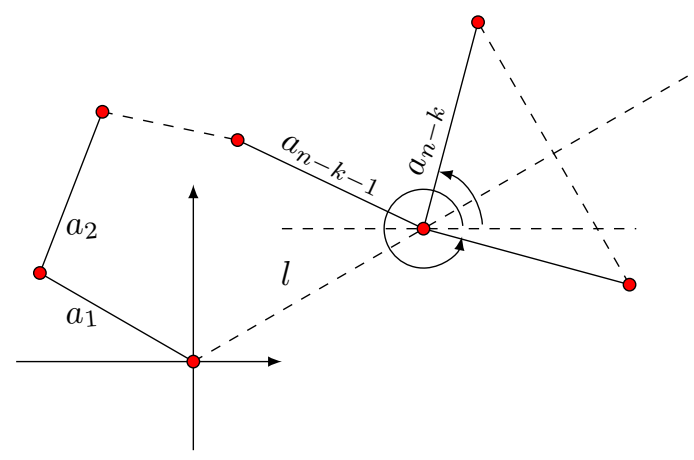

Figure 3. Choosing the value $\varepsilon_{n-k}$ in equation (3.6) corresponds to a flipping over the diagonal $l$.

a triangle orientation in [10] when building up a CKC from its diagonal lengths.

\section{Numerical simulations}

In this section we provide numerical examples that demonstrate the validity of the methods developed in this work. For illustrative purposes we will consider CKCs with five and six links. For CKSs with five links we will illustrate the spaces $\mathcal{S} \mathcal{D}_{a}$. We will compute random circular configurations for the CKCs by Theorem 2.1 and depict them in Figures 5 and 6. 


\subsection{CKCs with five and six links}

First we will consider a CKC with $n=5$ and $n=6$ links. The domain $\mathcal{S} \mathcal{D}_{a} \cap Q_{a}$ is depicted for two CKCs with different link lengths in Figure 4 . The black lines indicate the condition $-a_{1} a_{2}=C_{3}^{\min } \leq C_{3} \leq C_{3}^{\max }=a_{1} a_{2}$. The value $C_{4}$ lies within $\left[\max \left\{C_{4}^{\min }, C_{5}^{-}\right\}, \min \left\{C_{4}^{\max }, C_{5}^{+}\right\}\right]$. In the depicted cases $\max \left\{C_{4}^{\min }, C_{5}^{-}\right\}=C_{5}^{-}$and $\min \left\{C_{4}^{\max }, C_{5}^{+}\right\}=C_{5}^{+}$holds. Figure 5 shows 10 random circular configurations for CKCs with five links. Thereby the lengths of the last link is equal to the radius of the depicted circle. The configurations have been obtained for the orientation vector $\varepsilon^{4}=\left(\varepsilon_{2}, \varepsilon_{3}, \varepsilon_{4}\right)=(0,0,0)$.
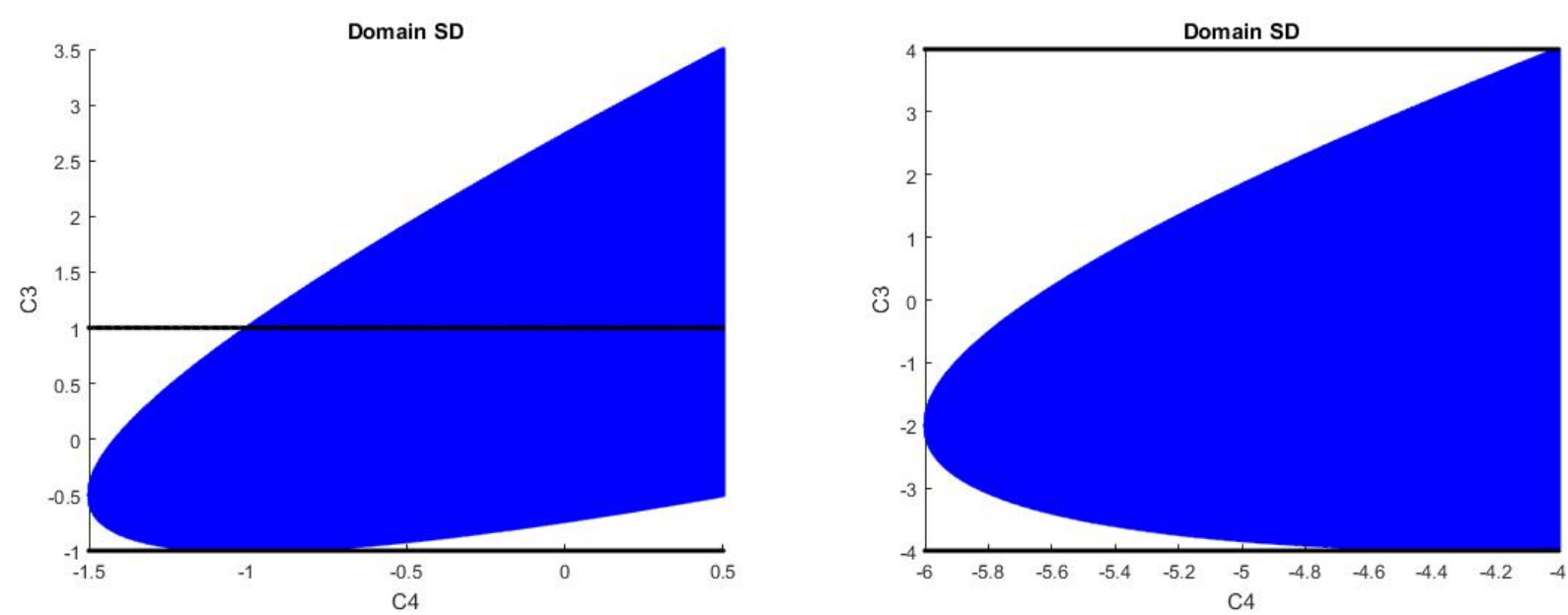

Figure 4. Domain $\mathcal{S D}_{a} \cap Q_{a}$ is the part of the blue area enclosed within the black lines. Left: CKC with all links equal to one. Right: CKC with link lengths $2,2,2,1,1$.
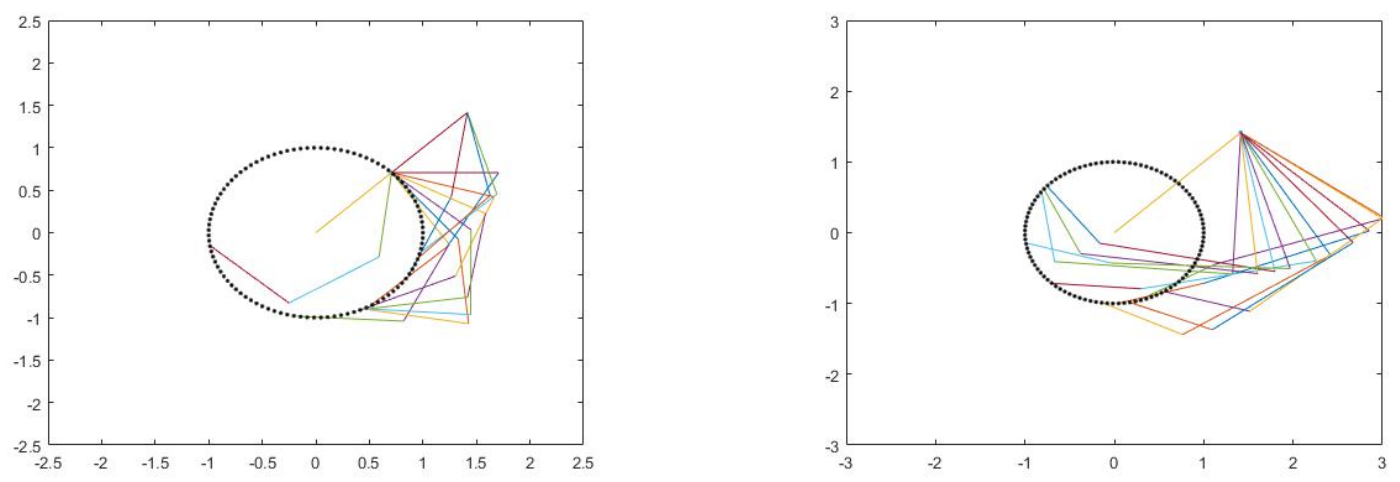

Figure 5. Circular configurations of two CKCs with five links. Left: Ten random circular configurations are depicted for the CKC with link lengths equal to one. Right: Ten random circular configurations are depicted for the CKC with link lengths $2,2,2,1,1$.

Finally, we give examples for CKSs with six links. Figure 6 shows random configurations for CKCs with six links. We consider the orientation vector $\varepsilon^{5}=\left(\varepsilon_{2}, \varepsilon_{3}, \varepsilon_{4}, \varepsilon_{5}\right)=(0,0,0,0)$.

\section{Conclusion and future work}

We have developed a new method to compute configurations in terms of joint angles of a CKC by a systematic procedure. Our approach does not require the solution of a system of linear inequalities by linear programming nor does it rely on probabilist methods. Numerical examples show validity of the proposed work. We expect that the described method can be useful in tasks like motion planning for CKCs. We expect that is an interesting line for future work to investigate the introduced map $\mathrm{P}^{a}: I^{n-3} \rightarrow \mathcal{S D \mathcal { U } _ { a }}$. We expect that it enables us to use tools from differential geometry, which may lead to further insights and applications. Moreover, it would be interesting to investigate how special designs for CKCs are reflected in the presented computations. 

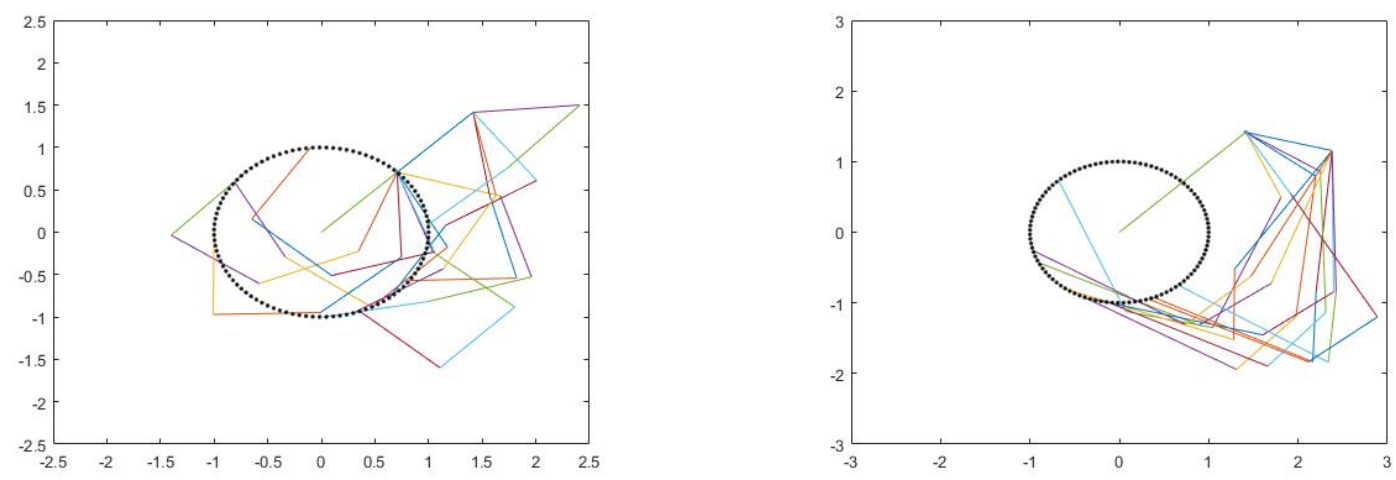

Figure 6. Circular configurations of two CKCs with six links. Left: Ten random circular configurations are depicted for the CKC with link lengths equal to one. Right: Ten random circular configurations are depicted for the CKC with link lengths $2,1,2,1,2,1$.

\section{A. Appendix}

The following Lemma guarantees that values obtained by the procedure described in section 2.2 are indeed real. We state the Lemma after the following definition:

Definition A.1. Furthermore, for $A \in \mathbb{R}$ let $\mathrm{E}_{k}(A)$ be the quadratic equation in the variable $C$ corresponding to the value $A$ given by

$$
(A-C)^{2}-a_{n-k}^{2}\left(S_{n-k-1}+2 C\right)=0
$$

and let $A^{ \pm}$be its solutions.

Lemma A.1. Let $C_{n-k+1}$ be a real number with $2 C_{n-k+1}+S_{n-k} \geq 0$, which means that the roots $C_{n-k+1}^{ \pm}$of the quadratic equation $\mathrm{E}_{k}\left(C_{n-k+1}\right)$, given by

$$
\left(C_{n-k+1}-C\right)^{2}-a_{n-k}^{2}\left(S_{n-k-1}+2 C\right)=0,
$$

are real. Choosing a value $C_{n-k}$ between the roots of $\mathrm{E}_{k}\left(C_{n-k+1}\right)$ guarantees that equation $\mathrm{E}_{k+1}\left(C_{n-k}\right)$ also has real solutions. Moreover, the roots of $\mathrm{E}_{1}\left(C_{n}\right)$ are real.

Proof. For $k=1$ we have that the roots $C_{n}^{ \pm}$of the equation $\mathrm{E}_{1}\left(C_{n}\right)$, given by

$$
\left(C_{n}-C\right)^{2}-a_{n-1}^{2}\left(S_{n-2}+2 C\right)=0,
$$

are real, since they are computed to be

$$
\begin{aligned}
C_{n}^{ \pm} & =C_{n}+a_{n-1}^{2} \pm a_{n-1} \sqrt{2 C_{n}+S_{n-1}} \\
& =\frac{a_{n}^{2}+a_{n-1}^{2}}{2}-\frac{S_{n-2}}{2} \pm a_{n-1} \sqrt{a_{n}^{2}-S_{n-1}+S_{n-1}} \\
& =\frac{a_{n}^{2}+a_{n-1}^{2}}{2}-\frac{S_{n-2}}{2} \pm a_{n-1} a_{n} .
\end{aligned}
$$

By our assumption the roots of $\mathrm{E}_{k}\left(C_{n-k+1}\right)$ are real. We have to prove that the roots of equation $\mathrm{E}_{k+1}\left(C_{n-k}\right)$ corresponding to $C_{n-k}$ are still real, whenever $C_{n-k+1}^{-} \leq C_{n-k} \leq C_{n-k+1}^{+}$. This means that the roots of

$$
\left(C_{n-k}-C\right)^{2}-a_{n-k-1}^{2}\left(S_{n-k-2}+2 C\right)=0
$$

are real, which is the case if $2 C_{n-k}+S_{n-k-1} \geq 0$. By our assumption $C_{n-k}$ is real and clearly $C_{n-k} \geq C_{n-k+1}^{-}$. Thus

$$
2 C_{n-k}+S_{n-k-1} \geq 2 C_{n-k+1}^{-}+S_{n-k-1} .
$$


The Lemma follows, if we can show that the right side of the last inequality is greater than zero. We plug in the explicit expression

$$
C_{n-k+1}^{-}=C_{n-k+1}+a_{n-k}^{2}-a_{n-k} \sqrt{2 C_{n-k+1}+S_{n-k}}
$$

into the latter inequality and obtain

$$
\begin{array}{r}
2\left(C_{n-k+1}+a_{n-k}^{2}-a_{n-k} \sqrt{2 C_{n-k+1}+S_{n-k}}\right)+S_{n-k-1} \\
2 C_{n-k+1}+\underbrace{\left(S_{n-k-1}+a_{n-k}^{2}\right)}_{=S_{n-k}}+a_{n-k}^{2}-2 a_{n-k} \sqrt{2 C_{n-k+1}+S_{n-k}} \geq 0 .
\end{array}
$$

By assumption $D:=2 C_{n-k+1}+S_{n-k} \geq 0$ and thus the latter inequality is satisfied since

$$
D+a_{n-k}^{2}-2 a_{n-k} \sqrt{D}=\left(\sqrt{D}-a_{n-k}\right)^{2} \geq 0 .
$$

As mentioned in section 2 the diagonal lengths of a CKC are closely related to the parameters $C_{n-k}$, which justifies the naming for $\mathcal{S D}_{a}$. More precisely, in [10] it is shown that $\beta^{n-1} \in \mathcal{C C}_{a}^{o n v}$ is a circular configuration if and only its diagonal lengths satisfy the system inequalities

$$
\left(L\left(\beta^{n-k}\right)-a_{n-k}\right)^{2} \leq L\left(\beta^{n-k-1}\right)^{2} \leq\left(L\left(\beta^{n-k}\right)+a_{n-k}\right)^{2},
$$

for $1 \leq k \leq n-2$. Note that here $L\left(\beta^{1}\right)=a_{1}$ and $L\left(\beta^{n-1}\right)=a_{n}$. Furthermore, it is reasonable here to consider circular configurations, since the diagonal lengths of a CKC are invariant with respect to rotation around the origin. The relation between the diagonal lengths an $\mathcal{S D _ { a }}$ is established by the lemma.

Lemma A.2. Let $\beta^{n-1} \in\left(S^{1}\right)^{n-1}$ be a vector of joint angles and let $C^{n-1}=\left(C_{3}, \ldots, C_{n-1}\right)$ be a vector, which entries are given by

$$
C_{n-k}:=X\left(\beta^{n-k-1}\right)
$$

for $1 \leq k \leq n-3$. Then $\beta^{n-1} \in \mathcal{C C}_{a}^{o n v}$ if and only if $C^{n-1} \in \mathcal{S D}_{a} \cap Q_{a}$.

Proof. Assume $C^{n-1} \in \mathcal{S D}_{a} \cap Q_{a}$ and $X\left(\beta^{n-k-1}\right)=C_{n-k}$ for a $\beta^{n-1} \in\left(S^{1}\right)^{n-1}$. Then, by (2.9) we have that the inequality $C_{n-k+1}^{-} \leq C_{n-k}$ is satisfied. Using the explicit expression (2.10) for $C_{n-k+1}^{-}$and our assumption we obtain that this is equivalent to

$$
C_{n-k+1}+a_{n-k}^{2}-a_{n-k} L\left(\beta^{n-k}\right) \leq C_{n-k} .
$$

Multiplying this inequality by two and then adding $S_{n-k-1}$ on both sides gives

$$
2 C_{n-k+1}+2 a_{n-k}^{2}-2 a_{n-k} L\left(\beta^{n-k}\right)+S_{n-k-1} \leq 2 C_{n-k}+S_{n-k-1}
$$

and therefore, since $S_{n-k-1}+a_{n-k}^{2}=S_{n-k}$, we have that

$$
\underbrace{2 C_{n-k+1}+S_{n-k}}_{=L\left(\beta^{n-k}\right)^{2}}+a_{n-k}^{2}-2 a_{n-k} L\left(\beta^{n-k}\right) \leq L\left(\beta^{n-k-1}\right)^{2} .
$$

Completing the square

$$
\left(L\left(\beta^{n-k}\right)-a_{n-k}\right)^{2} \leq L\left(\beta^{n-k-1}\right)^{2},
$$

shows that (A.4) is equivalent to the first inequality in (A.2). The second one follows from $C_{n-k} \leq C_{n-k+1}^{+}$ by analogous computations. Thus the system of inequalities (A.2) is satisfied and $\beta^{n-1} \in \mathcal{C C}_{a}^{\text {onv }}$. Conversely, if $\beta^{n-1} \in \mathcal{C C}_{a}^{o n v}$ is a circular configuration of a CKC its diagonals satisfy inequalities (A.2). Setting $C_{n-k}:=$ $X\left(\beta^{n-k-1}\right)$ and repeating the latter estimates shows that (2.9) are satisfied and thus $C^{n-1} \in \mathcal{S D}_{a} \cap Q_{a}$. 


\section{References}

[1] Suthakorn, J., Chirikjian, G.: A new inverse kinematics algorithm for binary manipulators with many actuators. Adv. Robotics 15 (2), $225-244$ (2000). https://doi.org/10.1163/15685530152116245

[2] Hinokuma, T., Shiga, H.: Topology of the Configuration Space of Polygons as a Codimension One of a Torus. Publ. RIMS, Kyoto Univ. 34 (4), 313-324 (1998). https:/ / doi.org/10.2977/prims/1195144628

[3] Hausmann, J. C., Knutson, A.: The cohomology ring of polygon spaces. Ann. Inst. Fourier 48 (1), $281-321$ (1998). https://doi.org/10.5802/aif.1619

[4] Cortes J., Simeon, T.:Sampling-based motion planning under kinematic loop closure constraints. In: Algorithmic Foundations of Robotics VI. Springer (2004). https://doi.org/10.1007/10991541_7

[5] Celaya E., Creemers, T., Ros, L.: Exact interval propagation for the efficient solution of position analysis problems on planar linkages. Mech. Mach. Theory 54, 116-131 (2012). https:/ / doi.org/10.1016/j.mechmachtheory.2012.03.005

[6] La Valle, S. M., Yakey, J. Kavraki, L.: A probabilistic roadmap approach for systems with closed kinemtic chains. In Proc. IEEE Int. Conf. Robot. Autom. (ICRA) 3, 1671-1676 (1999). https://10.1109/ROBOT.1999.770349

[7] La Valle, S. M.:Planing Algorithms. Cambridge University Press (2006).

[8] Han, L., Rudolph, L., Blumenthal, J., Valodzin, I.:Algorithmic Foundation of Robotics VII. Springer, 47, 235-250 (2008).

[9] Han, L., Rudolph, L.: Inverse Kinematics for a Serial Chain with Joints Under Distance Constraints. Robotics: Science and systems, 177-184 (2006).

[10] Han, L., Rudolph, L., Blumenthal, J., Valodzin, I.: Convexly stratified deformation spaces and efficient path planning for planar closed chains with revolute joints. Int. J. R. Res. 27, 1189-1212 (2008). https:// doi.org/10.1177/0278364908097211

[11] Milgram, R. J., Trinkle, J. C.: The geometry of configuration spaces for closed chains in two and three dimensions. Homol. Homotopy Appl. 6 (1), 237-267 (2004).

[12] Milgram, R. J., Trinkle, J. C.: Complete path planning for closed kinematic chains with spherical joints. Int. J. R. Res. 21 (9), $773-789$ (2002). https://doi.org/10.1177/0278364902021009119

[13] Jaillet, L., Porta, J. M.: Path planning under kinematic constraints by rapidly exploring manifolds. IEEE Trans. Robot. 29 (1), 105-117 (2012). https://doi.org/10.1109/TRO.2012.2222272

[14] Kapovich, M., Millson, J.: On the moduli spaces of polygons in the euclidean plane. J. Differential Geom. 42 (1), 133-164 (1995).

[15] Liu, G. F., Trinkle, J. C., Milgram, R. J.: Toward complete path planning for planar 3r-manipulators among point obstacles. Algorithmic Foundations of Robotics VI (2004).

[16] Luenberger D., Yinyu, Y.:Linear and nonlinear programming. Springer, (2004).

[17] Yakey, J. H., LaValle, S. M., Kavraki, L. E.: Randomized path planning for linkages with closed kinematic chains. IEEE Trans. Robot. Autom. 17 (6), 951-958 (2001). https://doi.org/10.1109/70.976030

[18] Yajia, Z., Hauser, K., Jingru L.: Robotics and Automation. (ICRA), 2013 IEEE International Conference on (2013).

\section{Affiliations}

\section{GERHARD ZANGERL}

AdDress: University of Innsbruck, Dept. of Mathematics, 6020, Innsbruck-Austria.

E-MAIL: Gerhard.Zangerl@uibk.ac.at

ORCID ID: 0000-0001-6145-4178 